ESAIM: ProceEdings, Vol. 4, 1998, 97-116

Contrôle et Équations aux DÉrivées Partielles

http://www.emath.fr/proc/Vol.4/

\title{
OPTIMAL DIRICHLET CONTROL AND INHOMOGENEOUS BOUNDARY VALUE PROBLEMS FOR THE UNSTEADY NAVIER-STOKES EQUATIONS
}

\author{
A. V. FURSIKOV ${ }^{1}$ \\ Department of Mechanics and Mathematics, Moscow \\ State University, Moscow 119899, Russia. \\ M. D. GUNZBURGER ${ }^{2}$ \\ Department of Mathematics and Statistics, York University, \\ North York, Ontario M3J 1P3, Canada; \\ Department of Mathematics, \\ Iowa State University, Ames, IA 50011-2064, USA. \\ L. S. $\mathrm{HOU}^{3}$ \\ Department of Mathematics and Statistics, York University, \\ North York, Ontario M3J 1P3, Canada; \\ Department of Mathematics, \\ Iowa State University, Ames, IA 50011-2064, USA.
}

Key Words : optimal control, Navier-Stokes equations, boundary value problem, drag minimization.

AMS Subject Qualification : 76D05,49J20,49K20,35K50.

\footnotetext{
${ }^{1}$ Supported in part by Department of Mathematics and International Institute of Theoretical and Applied Physics, Iowa State University while visiting Iowa State University, and by RFBI under Grant 960103550.

${ }^{2}$ Supported in part by the Natural Science and Engineering Research Council of Canada under Grant OGP-0137436.

${ }^{3}$ Supported in part by the Natural Science and Engineering Research Council of Canada under Grant OGP-0137436.
}

Article published by EDP Sciences and available at http://www.edpsciences.org/proc or http://dx.doi.org/10.1051/proc:1998023 


\begin{abstract}
We study optimal boundary control problems for the Navier-Stokes equations in an unbounded domain. The control is of Dirichlet type, i.e., the boundary velocity field. The drag functional is used as an example of control objectives. We identify the trace space for the velocity fields possessing finite energy, we prove the existence of a solution for the NavierStokes equations with boundary data belonging to the trace space, we establish the existence of an optimal solution over the control set, and we derive an optimality system of equations in the weak sense by using the Lagrange multiplier principles. The strong form of the optimality system of equations is also obtained and is described by a system of partial differential equations with boundary values.
\end{abstract}




\section{Introduction}

In this paper we consider general Dirichlet controls for the time-dependent Navier-Stokes system in the exterior of a bounded domain. Unsteady NavierStokes equations with distributed optimal control were investigated in [6],[7], $[15],[8]$. Boundary controls for steady-state Navier-Stokes equations were considered in [11], [12] and [13]. Boundary controls for time-dependent NavierStokes systems were considered in [3]-[5] wherein the control was assumed to be of the separation of variable type. General boundary controls for the twodimensional time-dependent Navier-Stokes systems were studied in [9]. In this paper, we present a procedure that applies to boundary controls for both the two-dimensional and three-dimensional Navier-Stokes equations.

The basic mathematical questions related to optimal boundary control problems are the well-posedness/existence of optimal solutions and the derivation of optimality system of equations. In order to answer these questions in the present situation of boundary controls for the Navier-Stokes equations, we need to resolve the inhomogeneous boundary value problems for the Navier-Stokes equations. The reasons for this are three-fold (in addition to the fact that such boundary value problems are important in their own right and such results were unavailable in the literature for the Navier-Stokes equations). First, in modelling the functional, one often needs to include a certain norm of the boundary control variable. The proper choice of such a norm depends on the knowledge of the boundary value problem, e.g., in what function spaces the boundary value problems are well-posed (so that the chosen norm makes sense). Second, to show the existence of an optimal solution, one needs a strong enough norm on the boundary control variable in the functional so as to be able to derive certain estimates for the velocity and pressure in the interior of the flow domain. Third, the derivation of the weak form of an optimality system by Lagrange multiplier rule essentially can be reduced to the solvability of a boundary value problem. We will resolve in this article inhomogeneous boundary value problems for the Navier-Stokes equations in both two- and three-dimensions, although the proofs are only given for the two-dimensional case. Of course, we will also answer the basic questions for the boundary control problem. In particular, we will derive the optimality system of equations in the weak form and also formulate the optimality system in a strong form.

The optimal control problems we consider can be described in general manner as follows: seek a boundary velocity field $\mathbf{g}$ and a corresponding velocitypressure pair $(\mathbf{v}, p)$ such that a functional

$$
\mathcal{J}(\mathbf{v}, \mathbf{g})=\mathcal{F}(\mathbf{v})+N \mathcal{G}(\mathbf{g})
$$

( $N$ is a positive constant) is minimized subject to the Navier-Stokes equations

$$
\begin{gathered}
\partial_{t} \mathbf{v}-\mu \Delta \mathbf{v}+\mathbf{v} \cdot \nabla \mathbf{v}+\nabla p=\mathbf{0} \quad \text { and } \quad \nabla \cdot \mathbf{v}=0 \quad \text { in }(0, T) \times \Omega, \\
\left.\mathbf{v}\right|_{t=0}=\mathbf{v}_{0} \quad \text { for } \mathbf{x} \in \Omega,\left.\quad \mathbf{v}\right|_{\partial \Omega}=\mathbf{g} \quad \text { for } t \in(0, T),
\end{gathered}
$$

ESAIM: Proc., Vol. 4, 1998, 97-116 
and

$$
\mathbf{v} \rightarrow \mathbf{v}_{\infty} \quad \text { as }|\mathbf{x}| \rightarrow \infty .
$$

Here, $\partial_{t}=\partial / \partial t, \Omega$ is the region exterior to a bounded body $B \subset \mathbb{R}^{d}(d=2,3)$ and $\partial \Omega$ is its boundary. For simplicity we assume $\partial \Omega$ is of class $C^{\infty}$ and is a simple connected closed surface. Also, $\mathbf{v}_{\infty}$ is a constant vector.

A control objective of importance in applications is the minimization of drag. We will use this objective in this paper to present our ideas. Many other control objectives can be handled with necessary modifications. The drag functional is briefly derived as follows. Let $\mathcal{T}=-p \mathcal{I}+2 \mu \mathcal{D}$ be the stress tensor, where $\mathcal{D}=\mathcal{D}(\mathbf{v})=\frac{1}{2}\left(\nabla \mathbf{v}+\nabla \mathbf{v}^{T}\right)$ is the rate of deformation tensor for the flow. The work needed to overcome the drag exerted on the given body $B=\mathbb{R}^{2} \backslash \Omega$ is given by

$$
\begin{aligned}
\mathcal{W} & =\int_{0}^{T} d t \int_{\partial \Omega}\left(\mathbf{v}-\mathbf{v}_{\infty}\right) \cdot(\mathcal{T} \mathbf{n}) d s \\
& =\int_{0}^{T} d t \int_{\partial \Omega}\left(\mathbf{v}-\mathbf{v}_{\infty}\right) \cdot\left\{-p \mathbf{n}+\mu\left(\nabla \mathbf{v}+\nabla \mathbf{v}^{T}\right) \mathbf{n}\right\} d s \\
& =\int_{0}^{T} d t \int_{\partial \Omega}\left(\mathbf{v}-\mathbf{v}_{\infty}\right) \cdot\left\{-p \mathbf{n}+\mu\left(\nabla\left(\mathbf{v}-\mathbf{v}_{\infty}\right)+\nabla\left(\mathbf{v}-\mathbf{v}_{\infty}\right)^{T}\right) \mathbf{n}\right\} d s .
\end{aligned}
$$

Upon setting $\mathbf{w}=\mathbf{v}-\mathbf{v}_{\infty}$, and using a Green's formula, we derive

$$
\mathcal{W}=\int_{0}^{T} \int_{\Omega} \mathbf{w} \cdot(\mu \Delta \mathbf{w}-\nabla p) d \mathbf{x} d t+2 \mu \int_{0}^{T} \int_{\Omega} \mathcal{D}(\mathbf{v}): \mathcal{D}(\mathbf{v}) d \mathbf{x} d t
$$

The Navier-Stokes equation for $\mathbf{v}$ yields the equation for $\mathbf{w}=\mathbf{v}-\mathbf{v}_{\infty}$ :

$$
\partial_{t} \mathbf{w}-\mu \Delta \mathbf{w}+\mathbf{v} \cdot \nabla \mathbf{w}+\mathbf{w} \cdot \nabla \mathbf{w}+\nabla p=\mathbf{0} .
$$

Combining the last two equations yields

$$
\begin{gathered}
\mathcal{W}=2 \mu \int_{0}^{T} d t \int_{\Omega} \mathcal{D}(\mathbf{w}): \mathcal{D}(\mathbf{w}) d \mathbf{x}+\frac{1}{2} \int_{0}^{T} d t \int_{\partial \Omega}|\mathbf{w}|^{2}\left(\mathbf{w}+\mathbf{v}_{\infty}\right) \cdot \mathbf{n} d s \\
+\frac{1}{2} \int_{\Omega}|\mathbf{w}(T, \mathbf{x})|^{2} d \mathbf{x}-\frac{1}{2} \int_{\Omega}|\mathbf{w}(0, \mathbf{x})|^{2} d \mathbf{x}
\end{gathered}
$$

The integral $\frac{1}{2} \int_{\Omega}|\mathbf{w}(t, \mathbf{x})|^{2} d \mathbf{x}$ is the (finite) kinetic energy of the difference flow $\mathbf{w}=\mathbf{v}-\mathbf{v}_{\infty}$. We can rewrite the last equation as the energy equality

$$
\begin{aligned}
& \mathcal{F}(\mathbf{w}) \equiv \frac{1}{4} \int_{\Omega}|\mathbf{w}(0, \mathbf{x})|^{2} d \mathbf{x}+\frac{\mathcal{W}}{2} \\
&=\frac{1}{4} \int_{\Omega}|\mathbf{w}(T, \mathbf{x})|^{2} d \mathbf{x}+\mu \int_{0}^{T} d t \int_{\Omega} \mathcal{D}(\mathbf{w}): \mathcal{D}(\mathbf{w}) d \mathbf{x} \\
& \quad+\frac{1}{4} \int_{0}^{T} d t \int_{\partial \Omega}|\mathbf{w}|^{2}\left(\mathbf{w}+\mathbf{v}_{\infty}\right) \cdot \mathbf{n} d s .
\end{aligned}
$$

Since the initial kinetic energy of the difference flow is given, it is quite natural to take the right-hand side of the last equation as the cost functional $\mathrm{F}(\mathbf{w})$ in terms of the difference flow $\mathbf{w}$ (for convenience, we introduce a factor of one-half).

For both physical and mathematical reasons, the size of the control should be constrained. Physically, one cannot realize controls of arbitrary size. Moreover, the cost of effecting control should be accounted for in the optimization process, e.g., one would not usually want to reduce the drag by a small amount if the cost of doing so is prohibitive. Limits on the size of the control are also needed 
in order to obtain a mathematically meaningful problem, e.g. to guarantee the existence of an optimal solution in a certain function class such that derivation of optimality system for considered problem should be possible.

We now discuss how we can choose a convenient functional $\mathcal{G}(\mathrm{g})$ from (1.1) for measuring the control. In order to apply Lagrange principle to derive the optimality system, we need to verify that the linearization, at an optimal solution, of the mapping defined by the constraints (i.e., the Navier-Stokes equations) is a surjective operator. This assertion can be proved in the space of sufficiently smooth functions where nonlinear term of the Navier-Stokes equations are subordinated to the linear terms. (This same condition also garantees the uniqueness of the Navier-Stokes solutions). It is well-known that in $2 D$ case the minimal level of smoothness for the velocity field $\mathbf{w}=\mathbf{v}-\mathbf{v}_{\infty}$ with the aforementioned properties is given by the space in which finite energy estimate holds, i.e. the space $\mathbf{w} \in L^{2}\left(0, T ; \mathbf{H}^{1}(\Omega)\right)$ and $\partial_{t} \mathbf{w} \in L^{2}\left(0, T ; \mathbf{H}^{-1}(\Omega)\right)$. In $3 D$ space it is the space $\mathbf{w} \in L^{2}\left(0, T ; \mathbf{H}^{3 / 2}(\Omega)\right)$ and $\partial_{t} \mathbf{w} \in L^{2}\left(0, T ; \mathbf{H}^{-1 / 2}(\Omega)\right)$. Note that these inclusions imply a certain behavior at infinity. (The Sobolev space notation used here is established in $\S 2$.) The boundary control should belong to a subset of the trace space on $\partial \Omega$ of the spaces described above for the vector field $\mathbf{w}$. The norm to be used as the functional $\mathcal{G}$ should be not weaker than the norm of the trace space. The precise choice of the norm will be given later.

\section{Functional spaces and statement of the opti- mal control problem.}

In order to state the optimal control problem mathematically, we need to introduce some functional spaces and notations. We will use the standard notations for the Lebesgue function space $L^{r}(\Omega)$ and the Sobolev spaces $H^{m}(\Omega), H^{l}(\partial \Omega)$ of sqare summarable functions for real smoothness indices $m, l$. For $m \geq 0$, we introduce the subspaces of the Sobolev spaces $H^{m}(\Omega)$

$$
H_{0}^{m}(\Omega)=\text { the closure of } C_{0}^{\infty}(\Omega) \text { in } H^{m}(\Omega)
$$

and the dual spaces $H^{-m}(\Omega)=\left(H_{0}^{m}(\Omega)\right)^{*}$ The vector counterparts of these spaces are denoted by $\mathbf{L}^{r}(\Omega), \mathbf{H}^{m}(\Omega), \mathbf{H}^{l}(\partial \Omega)$, and $\mathbf{H}_{0}^{m}(\Omega)$. For details, see [1] . We will also use the spaces of solenoidal vector fields

$$
\mathbf{V}^{m}(\Omega)=\left\{\mathbf{u} \in \mathbf{H}^{m}(\Omega): \nabla \cdot \mathbf{u}=0, \int_{\partial \Omega} \mathbf{u} \cdot \mathbf{n} d s=0\right\} \quad \text { for } m \geq 0
$$

and

$$
\mathbf{V}_{0}^{m}(\Omega)=\text { the closure of } \mathbf{C}_{0}^{\infty}(\Omega) \cap \mathbf{V}^{0}(\Omega) \text { in the } \mathbf{H}^{m}(\Omega) \text {-norm } \quad \text { for } m \geq 0,
$$

where when $m=0, \int_{\partial \Omega} \mathbf{u} \cdot \mathbf{n} d s$ is understood as the $H^{-1 / 2}(\partial \Omega)-H^{1 / 2}(\partial \Omega)$ duality pairing between the function $(\mathbf{u} \cdot \mathbf{n}) \in H^{-1 / 2}(\partial \Omega)$ and the constant 
scalar function $1 \in H^{1 / 2}(\partial \Omega)$. Note that for simplicity, we have assumed $\partial \Omega$ is a connected surface; otherwise, we need to require $\int_{\Gamma_{i}} \mathbf{u} \cdot \mathbf{n} d s=0$ on each connected component $\Gamma_{i}$ of $\partial \Omega$. Identifying $\left(\mathbf{V}_{0}^{0}(\Omega)\right)^{*}$ with $\mathbf{V}_{0}^{0}(\Omega)$ we introduce the dual spaces

$$
\mathbf{V}^{-m}(\Omega)=\left[\mathbf{V}_{0}^{m}(\Omega)\right]^{*} \quad \text { for } m \geq 1 .
$$

The norm on $\mathbf{V}^{m}(\Omega)$ and $\mathbf{V}_{0}^{m}(\Omega)$ are chosen to be that of $\mathbf{H}^{m}(\Omega)$. We also introduce the temporal-spatial function space, defined on $Q_{T}=(0, T) \times \Omega$,

$$
\mathcal{H}^{(s)}\left(Q_{T}\right)=\left\{f \in L^{2}\left(0, T ; H^{s}(\Omega)\right): \partial_{t} f \in L^{2}\left(0, T ; H^{s-2}(\Omega)\right)\right\}
$$

with norm

$$
\|f\|_{\mathcal{H}^{(s)}\left(Q_{T}\right)}^{2}=\|f\|_{L^{2}\left(0, T ; H^{s}(\Omega)\right)}^{2}+\left\|\partial_{t} f\right\|_{L^{2}\left(0, T ; H^{s-2}(\Omega)\right)}^{2},
$$

and the corresponding solenoidal function space

$$
\mathcal{V}^{(s)}\left(Q_{T}\right)=\left\{\mathbf{v} \in L^{2}\left(0, T ; \mathbf{V}^{s}(\Omega)\right): \partial_{t} \mathbf{v} \in L^{2}\left(0, T ; \mathbf{V}^{s-2}(\Omega)\right)\right\}
$$

with norm

$$
\|\mathbf{v}\|_{\mathcal{V}^{(s)}\left(Q_{T}\right)}^{2}=\|\mathbf{v}\|_{L^{2}\left(0, T ; \mathbf{V}^{s}(\Omega)\right)}^{2}+\left\|\partial_{t} \mathbf{v}\right\|_{L^{2}\left(0, T ; \mathbf{V}^{s-2}(\Omega)\right)}^{2} .
$$

Let us give the precise definition of a solution for the Navier-Stokes equations (in the $2 D$ case). Suppose that an initial condition $\mathbf{w}_{0} \in \mathbf{V}^{0}(\Omega)$ and a boundary condition

$$
\begin{gathered}
\mathbf{b} \in\left\{\mathbf{z} \in L^{2}\left(0, T ; \mathbf{H}^{1 / 2}(\Gamma)\right): \mathbf{z} \cdot \mathbf{n} \in H^{3 / 4}\left(0, T ; H^{-1}(\Gamma)\right),\right. \\
\left.\mathbf{z} \cdot \boldsymbol{\tau} \in H^{1 / 4}\left(0, T ; L^{2}(\Gamma)\right), \int_{\partial \Omega} \mathbf{z} \cdot \mathbf{n} d s=0\right\}
\end{gathered}
$$

are given where $\mathbf{n}$ is the outwards normal vector field along $\partial \Omega$ and $\boldsymbol{\tau}$ is the tangent vector field along $\partial \Omega$. Assume that $\mathbf{w}_{0}$ and $\mathbf{b}$ satisfy the compatibility condition

$$
\left.\mathbf{w} \cdot \mathbf{n}\right|_{\partial \Omega}=\left.\mathbf{b} \cdot \mathbf{n}\right|_{t=0} \quad \text { in } \quad \mathbf{H}^{-1 / 2}(\partial \Omega)
$$

The solution for the Navier-Stokes equations (1.2)-(1.4) is understood in the following sense (note that the decay of $\mathbf{w}=\mathbf{v}-\mathbf{v}_{\infty}$ at infinity follows automatically from the requirement $\left.\mathbf{w}(t) \in \mathcal{V}^{(1)}\left(Q_{T}\right)\right)$ :

Definition 2.1. $\mathbf{v}$ is said to be a solution of (1.2)-(1.4) if $\mathbf{v}=\mathbf{w}+\mathbf{v}_{\infty}$, where $\mathbf{w} \in \mathcal{V}^{(1)}\left(Q_{T}\right)$ satisfies

$$
\begin{aligned}
& \left\langle\partial_{t} \mathbf{w}(t), \mathbf{z}\right\rangle+2 \mu \int_{\Omega} \mathcal{D}(\mathbf{w}(t)): \mathcal{D}(\mathbf{z}) d \mathbf{x}+\int_{\Omega}(\mathbf{w}(t) \cdot \nabla) \mathbf{w}(t) \cdot \mathbf{z} d \mathbf{x} \\
& +\int_{\Omega}\left(\mathbf{v}_{\infty} \cdot \nabla\right) \mathbf{w}(t) \cdot \mathbf{z} d \mathbf{x}=0 \quad \forall \mathbf{z} \in \mathbf{V}_{0}^{1}(\Omega), \text { a.e. } t \in(0, T), \\
& \left.\quad \mathbf{w}\right|_{\partial \Omega}=\mathbf{b} \equiv \mathbf{g}-\mathbf{v}_{\infty} \quad \text { in } L^{2}\left(0, T ; \mathbf{H}^{1 / 2}(\partial \Omega)\right),
\end{aligned}
$$


and

$$
\left.\mathbf{w}\right|_{t=0}=\mathbf{w}_{0} \equiv \mathbf{v}_{0}-\mathbf{v}_{\infty} \quad \text { in } \mathbf{V}^{0}(\Omega) .
$$

In the $3 D$-case, the definition of a Navier-Stokes solution will be the same except that the space $\mathcal{V}^{(1)}\left(Q_{T}\right)$ is replaced by $\mathcal{V}^{(3 / 2)}\left(Q_{T}\right)$ (for solution $\mathbf{w}$ ), $\mathbf{V}^{0}(\Omega)$ relaced by $\mathbf{V}^{1 / 2}(\Omega)$ (for initial condition $\mathbf{w}_{0}$ ), and space (2.1) for a boundary condition $\mathbf{b}$ replaced by space (4.13) (see below).

The extremal problem we study is to minimize the drag functional $\mathcal{F}(\mathbf{w})$ derived in Section 1. It is more convenient to use the variable $\mathbf{w}=\mathbf{v}-\mathbf{v}_{\infty}$ rather than the variable $\mathbf{v}$. Also, we will simply use $\left.\mathbf{w}\right|_{\partial \Omega}$ to denote the Dirichlet control and, thus, will not introduce a separate notation $\mathbf{b}$ to denote the control variable and the boundary condition (2.4) will not be explicitly imposed as a constraint.

Thus the optimal control problem we consider can be stated as follows: seek a velocity field $\mathbf{w}$ such that the functional

$$
\begin{aligned}
& \mathcal{J}(\mathbf{w})=\mu \int_{0}^{T} \int_{\Omega} \mathcal{D}(\mathbf{w}): \mathcal{D}(\mathbf{w}) d \mathbf{x} d t \\
& \quad+\frac{1}{4} \int_{0}^{T} \int_{\partial \Omega}|\mathbf{w}|^{2}\left(\mathbf{w}+\mathbf{v}_{\infty}\right) \cdot \mathbf{n} d s d t+\frac{1}{4} \int_{\Omega}|\mathbf{w}(T, \mathbf{x})|^{2} d \mathbf{x}+N \mathcal{G}\left(\left.\mathbf{w}\right|_{\partial \Omega}\right)
\end{aligned}
$$

is minimized subject to the constraints (2.3) and (2.5).

\section{Boundary value problems and the optimality system}

Our eventual goal is to derive an optimality system of partial differential equations. This is achieved by using the Lagrange multiplier principles. This optimality system can serve as the basis for computing numerical approximations to optimal solutions. We will demonstrate in this section that the justification of the use of Lagrange multiplier rules can be reduced to the solvability of boundary value problems for a system similar to the linearized Navier-Stokes equations.

The abstract Lagrange multiplier rule is described as follows. Let $X_{1}$ and $X_{2}$ be two Banach spaces. Let $f: X_{1} \rightarrow \mathbb{R}$ be functional and $F: X_{1} \rightarrow X_{2}$ be a mapping. We seek a $w \in X_{1}$ such that

$$
f(w)=\inf _{u \in \mathcal{W}_{a d}} f(u), \quad \text { where } \quad \mathcal{W}_{a d}=\left\{u \in X_{1}: F(u)=0\right\} .
$$

The Lagrange functional for the minimization problem (3.1) is defined by

$$
\mathcal{L}(w, \boldsymbol{\lambda}, q)=\lambda f(w)+\langle F(w), q\rangle
$$

for all $w \in X_{1}, \lambda \in \mathbb{R}^{1}$ and $q \in X_{2}^{*}$. We quote a standard abstract Lagrange principle in the following particular form (see [2]).

ESAIM: Proc., Vol. 4, 1998, 97-116 
Theorem 3.1 Let $w$ be a solution of (3.1). Assume that the mappings $f$ and $F$ are continuously differentiable and that the image of the operator $F^{\prime}(w): X_{1} \rightarrow$ $X_{2}$ is closed. Then there exists $a q \in X_{2}^{*}$ and $a \lambda \in \mathbb{R}^{1}+$ such that the pair $(q, \lambda) \neq(0,0)$,

$$
\left\langle\mathcal{L}_{w}(w, \lambda, q), h\right\rangle=0 \quad \forall h \in X_{1},
$$

where $\mathcal{L}_{w}(\cdot, \cdot, \cdot)$ denotes the Fréchet derivative of $\mathcal{L}$ with respect to the first argument. Furthermore, if $F^{\prime}(w): X_{1} \rightarrow X_{2}$ is an epimorphism then $\lambda \neq 0$ and $\lambda$ can be taken as 1 .

To apply the abstract Lagrange principle to the optimal control problem stated in the previous section, we only need to verify the conditions that $\mathcal{G}$ is differentiable and $F^{\prime}(w)$ is an epimorphism. The differentiability of $\mathcal{G}$ can be easily met by choice (see Section 5). Thus the justification of the use of the Lagrange multiplier rule is reduced to showing that $F^{\prime}(w)$ is an epimorphism. To this end, we denote by $Y$ the appropriate space for the difference flow $\mathbf{w}$ (a concrete choice of $Y$ will be given in Section 5) and set $X_{1}=Y_{0}=\{\mathbf{y} \in Y$ : $\left.\left.\mathbf{y}\right|_{t=0}=\mathbf{0}\right\}$ and $X_{2}=L^{2}\left(0, T ; \mathbf{V}^{-1}(\Omega)\right)$. We define the mappings $f: X_{1} \rightarrow \mathbb{R}$ and $F: X_{1} \rightarrow X_{2}$ as follows:

$$
f(\mathbf{y})=\mathcal{J}(\mathbf{w}+\mathbf{y})
$$

and

$$
F(\mathbf{y})=\partial_{t}(\mathbf{w}+\mathbf{y})-\mu P \Delta(\mathbf{w}+\mathbf{y})+P\left[\left(\left(\mathbf{w}+\mathbf{y}+\mathbf{v}_{\infty}\right) \cdot \nabla\right)(\mathbf{w}+\mathbf{y})\right]
$$

where $P: \mathbf{H}^{-1}(\Omega) \rightarrow \mathbf{V}^{-1}(\Omega)$ is the projection operator. Then $\mathbf{y}=0$ is the solution of the correspondent extremal problem and $F^{\prime}(\mathbf{0}): X_{1} \rightarrow X_{2}$ is defined by

$$
\left\langle F^{\prime}(\mathbf{0}), \mathbf{y}\right\rangle=\partial_{t} \mathbf{y}-\mu P \Delta \mathbf{y}+P\left[(\mathbf{y} \cdot \nabla) \mathbf{w}+\left(\left(\mathbf{w}+\mathbf{v}_{\infty}\right) \cdot \nabla\right) \mathbf{y}\right] .
$$

To show $F^{\prime}(\mathbf{0})$ is an epimorphism, we first observe that this operator is continuous. Next we need to show that for each $\mathbf{f} \in L^{2}\left(0, T ; \mathbf{V}^{-1}(\Omega)\right)$ the system

$$
\begin{aligned}
& \left\langle\partial_{t} \mathbf{y}(t), \mathbf{z}\right\rangle+\mu \int_{\Omega} \nabla \mathbf{y}(t): \nabla \mathbf{z} d \mathbf{x}+\int_{\Omega}\left(\left(\mathbf{w}(t)+\mathbf{v}_{\infty}\right) \cdot \nabla\right) \mathbf{y}(t) \cdot \mathbf{z} d \mathbf{x} \\
& \quad+\int_{\Omega}(\mathbf{y}(t) \cdot \nabla) \mathbf{w}(t) \cdot \mathbf{z} d \mathbf{x}=\int_{\Omega} \mathbf{f}(t) \cdot \mathbf{z} d \mathbf{x} \quad \forall \mathbf{z} \in \mathbf{V}_{0}^{1}(\Omega), \text { a.e. } t \in(0, T),
\end{aligned}
$$

and

$$
\left.\mathbf{y}\right|_{t=0}=\mathbf{0} \quad \text { in } \mathbf{V}^{0}(\Omega)
$$

has a solution $\mathbf{y} \in Y_{0}$. We can supplement this system with a boundary condition, e.g.,

$$
\left.\mathbf{y}\right|_{(0, T) \times \partial \Omega}=\mathbf{0} .
$$

Thus we see that the derivation of the optimality system by the Lagrange multiplier rule is reduced to the solvability of the boundary value problem (3.4)-(3.6). 
This system of differential equations is similar to a system of linearized NavierStokes equations with coefficients $\mathbf{w}(t)$. To prove the solvability of $(3.4)-(3.6)$ we need the optimal solution $\mathbf{w}(t)$ (which coincides with the coefficient $\mathbf{w}(t)$ in $(3.4))$ to be smooth enough. To this end, we need to consider the existence theorem for the optimal control problem in a class of sufficiently smooth functions. Thus, the derivation of the optimality system is reduced to the solvability theorem for inhomogeneous boundary value problems for the Navier-Stokes equations in a class of sufficiently smooth vector fields. The question of the solvability of this boundary value problem can be answered using the techniques discussed in the next section (Section 4.2).

\section{Trace theorems and solutions of the Navier- Stokes equations}

As has been repeatedly pointed out in this article, the study of Dirichlet boundary value problems for the Navier-Stokes equations is of paramount importance in the study of boundary controls for the Navier-Stokes equations. We devote this section to the study of boundary value problems.

Based on the well-known results regarding the existence and uniqueness of solutions for the Navier-Stokes equations with homogeneous Dirichlet conditions, we should seek solutions of the inhomogeneous Dirichlet problem in the spaces $\mathcal{V}^{(1)}\left(Q_{T}\right)=L^{2}\left(0, T ; \mathbf{V}^{1}(\Omega)\right) \cap H^{1}\left(0, T ; \mathbf{V}^{-1}(\Omega)\right)$ in $2 D$ and $\mathcal{V}^{(3 / 2)}\left(Q_{T}\right)=$ $L^{2}\left(0, T ; \mathbf{V}^{3 / 2}(\Omega)\right) \cap H^{1}\left(0, T ; \mathbf{V}^{-1 / 2}(\Omega)\right)$ in $3 D$. Thus, the Dirichlet boundary conditions should be imposed in the trace spaces of $\mathcal{V}^{(1)}\left(Q_{T}\right)$ in $2 D$ and $\mathcal{V}^{(3 / 2)}\left(Q_{T}\right)$ in $3 D$, respectively. We will consider the trace problems in Section 4.1. The proofs are only given for the $2 D$ case. Then in Section 4.2 we discuss solutions of the Navier-Stokes equations with inhomogeneous boundary conditions.

\subsection{Trace theorems.}

In the $2 D$ case, we have the precise trace results, i.e., the trace space of

$$
\mathcal{V}^{(1)}\left(Q_{T}\right)=L^{2}\left(0, T ; \mathbf{V}^{1}(\Omega)\right) \cap H^{1}\left(0, T ; \mathbf{V}^{-1}(\Omega)\right) \quad(\text { in } 2 D)
$$

is characterized as follows:

$$
\begin{gathered}
\operatorname{tr}\left[\mathcal{V}^{(1)}\left(Q_{T}\right)\right]=\left\{\mathbf{z} \in L^{2}\left(0, T ; \mathbf{H}^{1 / 2}(\partial \Omega)\right): \mathbf{z} \cdot \mathbf{n} \in H^{3 / 4}\left(0, T ; H^{-1}(\partial \Omega)\right)\right. \\
\left.\mathbf{z} \cdot \boldsymbol{\tau} \in H^{1 / 4}\left(0, T ; L^{2}(\partial \Omega)\right), \int_{\partial \Omega} \mathbf{z} \cdot \mathbf{n} d s=0\right\}
\end{gathered}
$$

The trace results is a local property (local to the boundary $\partial \Omega$ ). Thus we only need to consider the trace results for functions with support in a neighborhood of $\partial \Omega$. We first examine the necessary condition of the trace theorem. The proofs are only given for the $2 D$ case.

ESAIM: Proc., Vol. 4, 1998, 97-116 
Theorem 4.1. Assume that $\Omega \subset \mathbb{R}^{2}$ and $\mathbf{u} \in \mathcal{V}^{(1)}\left(Q_{T}\right)$ with a compact support in its spatial variables. Then

$$
\begin{gathered}
\left.\mathbf{u}\right|_{\partial \Omega} \in\left\{\mathbf{z} \in L^{2}\left(0, T ; \mathbf{H}^{1 / 2}(\partial \Omega)\right): \mathbf{z} \cdot \mathbf{n} \in H^{3 / 4}\left(0, T ; H^{-1}(\partial \Omega)\right),\right. \\
\left.\mathbf{z} \cdot \boldsymbol{\tau} \in H^{1 / 4}\left(0, T ; L^{2}(\partial \Omega)\right), \int_{\partial \Omega} \mathbf{z} \cdot \mathbf{n} d s=0\right\} .
\end{gathered}
$$

Furthermore,

$$
\begin{aligned}
\left(\left\|\left.\mathbf{u}\right|_{\partial \Omega}\right\|_{L^{2}\left(0, T ; \mathbf{H}^{1 / 2}(\partial \Omega)\right)}^{2}+\left\|\left.\mathbf{u} \cdot \mathbf{n}\right|_{\partial \Omega}\right\|_{H^{3 / 4}\left(0, T ; H^{-1}(\partial \Omega)\right)}^{2}+\left\|\left.\mathbf{u} \cdot \boldsymbol{\tau}\right|_{\partial \Omega}\right\|_{H^{1 / 4}\left(0, T ; L^{2}(\partial \Omega)\right)}^{2}\right) . . & . . \leq C\|\mathbf{u}\|_{\mathcal{V}^{(1)}\left(Q_{T}\right)}^{2} \\
& \text { where } C \text { is a constant independent of } \mathbf{u} .
\end{aligned}
$$

Proof. We first note that the well-known trace result $\operatorname{tr}\left(\mathbf{H}^{1}(\Omega)\right)=\mathbf{H}^{1 / 2}(\partial \Omega)$ implies

$$
\left\|\left.\mathbf{u}\right|_{\partial \Omega}\right\|_{L^{2}\left(0, T ; \mathbf{H}^{1 / 2}(\partial \Omega)\right)}^{2} \leq C\|\mathbf{u}\|_{L^{2}\left(0, T ; \mathbf{H}^{1}(\Omega)\right)}^{2} .
$$

Using the divergence free condition of $\mathbf{u}$, we can find a stream function $\psi \in$ $H^{1}(\Omega)$ such that

$$
\frac{\partial \psi}{\partial x_{2}}=u_{1} \quad \text { and } \quad \frac{\partial \psi}{\partial x_{1}}=-u_{2}
$$

see, e.g., [10]. We denote by $\boldsymbol{\tau}=\left(\tau_{1}, \tau_{2}\right)^{T}$ and $\mathbf{n}=\left(n_{1}, n_{2}\right)^{T}$ the unit counterclockwise tangent and outward normal vectors, respectively, along $\partial \Omega$. We have the following relations:

$$
\tau_{1}=n_{2} \quad \text { and } \quad \tau_{2}=-n_{1} .
$$

It can be easily verified that

$$
\left.(\mathbf{u} \cdot \mathbf{n})\right|_{\partial \Omega}=-\left.\left(\partial_{\tau} \psi\right)\right|_{\partial \Omega} \quad \text { and }\left.\quad(\mathbf{u} \cdot \boldsymbol{\tau})\right|_{\partial \Omega}=\left.\left(\partial_{n} \psi\right)\right|_{\partial \Omega}
$$

where $\partial_{\tau} \psi$ and $\partial_{n} \psi$ are the tangential and normal derivatives of $\psi$, respectively. Thus the proof of the lemma boils down to checking the regularity of $\left.\left(\partial_{\tau} \psi\right)\right|_{\partial \Omega}$ and $\left.\left(\partial_{n} \psi\right)\right|_{\partial \Omega}$. Taking the $t$-derivatives of (4.1) we have

$$
\frac{\partial \psi_{t}}{\partial x_{2}}=\left(u_{1}\right)_{t} \quad \text { and } \quad \frac{\partial \psi_{t}}{\partial x_{1}}=-\left(u_{2}\right)_{t}
$$

where $(\cdot)_{t}$ stands for the partial derivative with respect to $t$. Using the regularity $\mathbf{u} \in L^{2}\left(0, T ; \mathbf{H}^{1}(\Omega)\right)$ and $\mathbf{u}_{t} \in L^{2}\left(0, T ; \mathbf{H}^{-1}(\Omega)\right)$, we deduce that (see [10])

$$
\psi \in \mathcal{H}^{(2)}((0, T) \times \Omega)=\left\{\phi \in L^{2}\left(0, T ; H^{2}(\Omega)\right): \phi_{t} \in L^{2}\left(0, T ; L^{2}(\Omega)\right)\right\} .
$$

$\partial \Omega$ being of class $C^{\infty}$, we may choose a neighborhood $U$ of $\partial \Omega$ and an orthonormal coordinate system $\left(x_{1}^{\prime}, x_{2}^{\prime}\right)^{T}$ such that $U=\left\{\mathbf{x}=\left(x_{1}^{\prime}, x_{2}^{\prime}\right)^{T}:\left(x_{1}^{\prime}, 0\right)^{T} \in\right.$ $\left.\partial \Omega, x_{2}^{\prime} \in[0, \epsilon]\right\}$ for some $\epsilon>0$ and that along $\partial \Omega$, this new coordinate system 
coincides with the tangential-normal coordinate system. The space $\mathcal{H}^{(2)}((0, T) \times$ $U$ ) can be rewritten in the form

$$
\begin{aligned}
& \mathcal{H}^{(2)}((0, T) \times U) \\
& =\left\{\psi\left(x_{2}^{\prime}, t, x_{1}^{\prime}\right) \in L^{2}\left(0, \epsilon ; L^{2}\left(0, T ; H^{2}(\partial \Omega)\right)\right) \cap L^{2}\left(0, \epsilon ; H^{1}\left(0, T ; L^{2}(\partial \Omega)\right)\right):\right. \\
& \left.\quad \partial_{x_{2}^{\prime} x_{2}^{\prime}} \psi \in L^{2}\left(0, \epsilon ; L^{2}\left(0, T ; L^{2}(\partial \Omega)\right)\right)\right\} .
\end{aligned}
$$

By virtue of a trace theorem of [16], we have that the mappings $\gamma_{0}:\left.\psi \mapsto \psi\right|_{x_{2}^{\prime}=0}$ and $\gamma_{1}:\left.\psi \mapsto \partial_{x_{2}^{\prime}} \psi\right|_{x_{2}^{\prime}=0}$ are well-defined on $\mathcal{H}^{(2)}((0, T) \times U)$; furthermore, the mapping $\psi \mapsto\left(\gamma_{0} \psi, \gamma_{1} \psi\right)$ from $\mathcal{H}^{(2)}((0, T) \times U)$ to

$$
\begin{aligned}
& {\left[L^{2}\left(0, T ; H^{2}(\partial \Omega)\right) \cap H^{1}\left(0, T ; L^{2}(\partial \Omega)\right), L^{2}\left(0, T ; L^{2}(\partial \Omega)\right)\right]_{3 / 4}} \\
& \times\left[L^{2}\left(0, T ; H^{2}(\partial \Omega)\right) \cap H^{1}\left(0, T ; L^{2}(\partial \Omega)\right), L^{2}\left(0, T ; L^{2}(\partial \Omega)\right)\right]_{1 / 4}
\end{aligned}
$$

is continuous and surjective. Here we have used the intermediate spaces $[\mathcal{X}, \mathcal{Y}]_{\alpha}$, $\alpha \in[0,1]$, of the Hilbert spaces $\mathcal{X}$ and $\mathcal{Y}$ as defined in [16]. Using the definition of these intermediate spaces (see [16]), we obtain

$$
\begin{aligned}
& {\left[L^{2}\left(0, T ; H^{2}(\partial \Omega)\right) \cap H^{1}\left(0, T ; L^{2}(\partial \Omega)\right), L^{2}\left(0, T ; L^{2}(\partial \Omega)\right)\right]_{3 / 4}} \\
& \quad=L^{2}\left(0, T ; H^{3 / 2}(\partial \Omega)\right) \cap H^{3 / 4}\left(0, T ; L^{2}(\partial \Omega)\right)
\end{aligned}
$$

and

$$
\begin{aligned}
& {\left[L^{2}\left(0, T ; H^{2}(\partial \Omega)\right) \cap H^{1}\left(0, T ; L^{2}(\partial \Omega)\right), L^{2}\left(0, T ; L^{2}(\partial \Omega)\right)\right]_{1 / 4}} \\
& \quad=L^{2}\left(0, T ; H^{1 / 2}(\partial \Omega)\right) \cap H^{1 / 4}\left(0, T ; L^{2}(\partial \Omega)\right) .
\end{aligned}
$$

Hence the mapping $\psi \mapsto\left(\gamma_{0} \psi, \gamma_{1} \psi\right)$ is continuous and surjective from $\mathcal{H}^{(2)}((0, T) \times U)$ to

$$
\begin{aligned}
& {\left[L^{2}\left(0, T ; H^{3 / 2}(\partial \Omega)\right) \cap H^{3 / 4}\left(0, T ; L^{2}(\partial \Omega)\right)\right]} \\
& \times\left[L^{2}\left(0, T ; H^{1 / 2}(\partial \Omega)\right) \cap H^{1 / 4}\left(0, T ; L^{2}(\partial \Omega)\right)\right],
\end{aligned}
$$

i.e., we have shown that

$$
\left.\psi\right|_{\partial \Omega} \in H^{3 / 4}\left(0, T ; L^{2}(\partial \Omega)\right) \quad \text { and }\left.\quad \partial_{n} \psi\right|_{\partial \Omega} \in H^{1 / 4}\left(0, T ; L^{2}(\partial \Omega)\right)
$$

with the estimates

$$
\begin{aligned}
\left\|\left.\psi\right|_{\partial \Omega}\right\|_{H^{3 / 4}\left(0, T ; L^{2}(\partial \Omega)\right)} & \leq C\|\psi\|_{\mathcal{H}^{(2)}((0, T) \times \Omega)} \leq C\|\mathbf{u}\|_{\mathcal{V}^{(1)}((0, T) \times \Omega)} \\
\left\|\left.\partial_{n} \psi\right|_{\partial \Omega}\right\|_{H^{1 / 4}\left(0, T ; L^{2}(\partial \Omega)\right)} & \leq C\|\psi\|_{\mathcal{H}^{(2)}((0, T) \times \Omega)} \leq C\|\mathbf{u}\|_{\mathcal{V}^{(1)}((0, T) \times \Omega)}
\end{aligned}
$$

where the positive constant $C$ is indepndent of $\psi$ and $\mathbf{u}$. Using relation (4.2) we easily see that $\mathbf{u} \cdot \mathbf{n} \in H^{3 / 4}\left(0, T ; H^{-1}(\partial \Omega)\right)$ and $\mathbf{u} \cdot \boldsymbol{\tau} \in H^{1 / 4}\left(0, T ; L^{2}(\partial \Omega)\right)$ satisfying the desired estimate.

To prove the sufficient condition of the trace results, i.e., the extension theorem, we first prove the following lemma.

ESAIM: Proc., Vol. 4, 1998, 97-116 
Lemma 4.2. Assume that a pair of functions $\left(b_{\tau}, h\right)$ defined on $S_{T}=(0, T) \times \partial \Omega$ satisfies

$$
b_{\tau} \in L^{2}\left(0, T ; H^{1 / 2}(\partial \Omega)\right) \cap H^{1 / 4}\left(0, T ; L^{2}(\partial \Omega)\right)
$$

and

$$
h \in L^{2}\left(0, T ; H^{3 / 2}(\partial \Omega)\right) \cap H^{3 / 4}\left(0, T ; L^{2}(\partial \Omega)\right) .
$$

Then there exists an extension $\psi \in \mathcal{H}^{(2)}\left(Q_{T}\right)$ satisfying

$$
\begin{gathered}
\left.\left(\partial_{n} \psi\right)\right|_{S_{T}}=b_{\tau},\left.\quad\left(\partial_{n} \psi\right)\right|_{S_{T}}=h, \\
\|\psi\|_{\mathcal{H}^{(2)}\left(Q_{T}\right)}^{2} \leq C\left\{\begin{array}{l}
\left\|b_{\tau}\right\|_{L^{2}\left(0, T ; H^{1 / 2}(\partial \Omega)\right)}^{2}+\left\|b_{\tau}\right\|_{H^{1 / 4}\left(0, T ; L^{2}(\partial \Omega)\right)}^{2} \\
+\|h\|_{L^{2}\left(0, T ; H^{3 / 2}(\partial \Omega)\right)}^{2}+\|h\|_{H^{3 / 4}\left(0, T ; L^{2}(\partial \Omega)\right)}^{2}
\end{array}\right\},
\end{gathered}
$$

and

$$
\psi \text { vanishes outside a neighborhood of } S_{T}=(0, T) \times \partial \Omega,
$$

where $C$ is a constant independent of $b_{\tau}, h$, and $\psi$.

Proof. As was indicated in the proof of the Theorem 4.1, the mapping $\psi \mapsto$ $\left(\left.\psi\right|_{S_{T}},\left.\left(\partial_{n} \psi\right)\right|_{S_{T}}\right)$ is continuous and surjective from $\mathcal{H}^{(2)}((0, T) \times U)$ to

$$
\begin{aligned}
& {\left[L^{2}\left((0, T) ; H^{1 / 2}(\partial \Omega)\right) \cap H^{1 / 4}\left((0, T) ; L^{2}(\partial \Omega)\right)\right] \times\left[L^{2}\left((0, T) ; H^{3 / 2}(\partial \Omega)\right)\right.} \\
&\left.\cap H^{3 / 4}\left((0, T) ; L^{2}(\partial \Omega)\right)\right] .
\end{aligned}
$$

As in Theorem 4.1 proof we may choose a neighborhood $U$ of $\partial \Omega$ and an tangential-normal coordinate system $\left(x_{1}^{\prime}, x_{2}^{\prime}\right)^{T}$ such that $U=\left\{\mathbf{x}=\left(x_{1}^{\prime}, x_{2}^{\prime}\right)^{T}\right.$ : $\left.\left(x_{1}^{\prime}, 0\right)^{T} \in \partial \Omega, x_{2}^{\prime} \in[0, \epsilon]\right\}$ for some $\epsilon>0$. Hence, by the trace theorem from [16] given $b_{\tau}$ and $h$ satisfying (4.3)-(4.4), there exists a $\psi$ satisfying (4.5) and the estimate

$$
\|\psi\|_{\mathcal{H}^{(2)}((0, T) \times U)} \leq C\left\{\begin{array}{l}
\left\|b_{\tau}\right\|_{L^{2}\left(0, T ; H^{1 / 2}(\partial \Omega)\right)}+\left\|b_{\tau}\right\|_{H^{1 / 4}\left(0, T ; L^{2}(\partial \Omega)\right)} \\
\left.+\|h\|_{L^{2}\left(0, T ; H^{3 / 2}(\partial \Omega)\right)}+\|h\|_{H^{3 / 4}\left(0, T ; L^{2}(\partial \Omega)\right)}\right\},
\end{array}\right.
$$

where $C$ is independent of $\psi, b_{\tau}$ and $h$. Finally, we may choose another neighborhood $\widetilde{U}$ of $(0, \varepsilon) \times \partial \Omega$ such that the closure of $U$ is contained in $\widetilde{U}$. Well-known extension results allow us to extend continuously the space $\mathcal{H}^{(2)}((0, T) \times U)$ into the space $\left\{\phi \in \mathcal{H}^{(2)}((0, T) \times \Omega): \phi\right.$ vanishes outside $\left.\widetilde{U}\right\}$.

We are now in a position to prove the extension result.

Theorem 4.3. Assume that $b_{n}$ and $b_{\tau}$ satisfy

$$
\int_{\partial \Omega} b_{n} d s=0 \quad \text { a.e. } t \in[0, T]
$$




$$
b_{n} \in L^{2}\left(0, T ; H^{1 / 2}(\partial \Omega)\right) \cap H^{3 / 4}\left(0, T ; H^{-1}(\partial \Omega)\right),
$$

and

$$
b_{\tau} \in L^{2}\left(0, T ; H^{1 / 2}(\partial \Omega)\right) \cap H^{1 / 4}\left(0, T ; L^{2}(\partial \Omega)\right) .
$$

Then, there exists $a \mathbf{u} \in \mathcal{V}^{(1)}\left(Q_{T}\right)$ satisfying

$$
\left.\mathbf{u}\right|_{S_{T}}=\mathbf{b} \equiv b_{n} \mathbf{n}+b_{\tau} \boldsymbol{\tau}
$$

and the estimate

$\|\mathbf{u}\|_{\mathcal{V}^{(1)}\left(Q_{T}\right)}^{2} \leq C\left\{\|b\|_{L^{2}\left(0, T ; H^{1 / 2}(\partial \Omega)\right.}^{2}+\left\|b_{n}\right\|_{H^{3 / 4}\left(0, T ; H^{-1}(\partial \Omega)\right)}^{2}+\left\|b_{\tau}\right\|_{H^{1 / 4}\left(0, T ; L^{2}(\partial \Omega)\right)}^{2}\right\}$,

where $C$ is a constant independent of $b_{n}$ and $b_{\tau}$, and such that $\mathbf{u}$ vanishes outside a neighborhood of $(0, T) \times \partial \Omega$.

Proof. We define

$$
h(t, \mathbf{x})=-\int_{\mathbf{x}_{0}}^{\mathbf{x}} b_{n}(t, \mathbf{x}(s)) d s \quad \forall \mathbf{x} \in \partial \Omega,
$$

where the line integral on the right-hand side is taken counter-clockwise along $\partial \Omega$ starting from a fixed point $\mathbf{x}_{0} \in \partial \Omega$. Evidently, $h \in L^{2}\left((0, T) ; H^{3 / 2}(\partial \Omega)\right) \cap$ $H^{3 / 4}\left((0, T) ; L^{2}(\partial \Omega)\right)$. Then, Lemma 4.2 implies that there exists an $\psi \in$ $\mathcal{H}^{(2)}\left(Q_{T}\right)$ which vanishes outside a neighborhood of $(0, T) \times \partial \Omega$ such that

$$
\left.\psi\right|_{S_{T}}=h \quad \text { and }\left.\quad \partial_{n} \psi\right|_{S_{T}}=b_{\tau} .
$$

By setting

$$
\mathbf{u}=\operatorname{curl} \psi=\left(\begin{array}{c}
\partial_{x_{2}} \psi \\
-\partial_{x_{1}} \psi
\end{array}\right)
$$

we see that

$$
\begin{gathered}
\mathbf{u} \in \mathcal{V}^{(1)}\left(Q_{T}\right), \\
\left.(\mathbf{u} \cdot \mathbf{n})\right|_{S_{T}}=\left.(\operatorname{curl} \psi \cdot \mathbf{n})\right|_{S_{T}}=-\nabla \psi \cdot \boldsymbol{\tau}=-\partial_{\tau} \psi=-\partial_{\tau} h=b_{n},
\end{gathered}
$$

and

$$
\left.(\mathbf{u} \cdot \boldsymbol{\tau})\right|_{S_{T}}=\left.(\operatorname{curl} \psi \cdot \boldsymbol{\tau})\right|_{S_{T}}=\nabla \psi \cdot \mathbf{n}=\partial_{n} \psi=b_{\tau} .
$$

Estimate (4.12) follows from the previous lemma.

Now we formulate the restriction and extension results for the space $\mathcal{V}^{(3 / 2)}\left(Q_{T}\right)$ in the $3 D$-case. Every vector field $\mathbf{u}(x)$ defined on $\partial \Omega$ can be decomposed as follows:

$$
\mathbf{u}(x)=u_{n}(x) \mathbf{n}(x)+\mathbf{u}_{\boldsymbol{\tau}}(x)
$$

where $\mathbf{n}(x)$ is the outward normal to $\partial \Omega, u_{n}$ is the projection of $\mathbf{u}(x)$ in $\mathbf{n}(x)$ direction, and $\mathbf{u}_{\boldsymbol{\tau}} \in T_{x} \partial \Omega$ is the tangential component of $u(x)$ along $\partial \Omega$. These notations will be used below.

ESAIM: Proc., Vol. 4, 1998, 97-116 
Theorem 4.4 Assume that

$$
\mathbf{u} \in \mathcal{V}^{(3 / 2)}\left(Q_{T}\right)=L^{2}\left(0, T ; \mathbf{V}^{3 / 2}(\Omega)\right) \cap H^{1}\left(0, T ; \mathbf{V}^{-1 / 2}(\Omega)\right) .
$$

Then for every $\varepsilon>0$,

$$
\begin{aligned}
\left.\mathbf{u}\right|_{\partial \Omega} \in\{ & \mathbf{z} \in L^{2}\left(0, T ; \mathbf{H}^{1}(\partial \Omega)\right): b_{n} \in H^{1-\varepsilon}\left(0, T ; \mathbf{H}^{-1}(\partial \Omega)\right), \\
& \mathbf{z} \boldsymbol{\tau} \in H^{1 / 2}\left(0, T ; \mathbf{L}_{2}(\partial \Omega), \quad \int_{\partial \Omega} z_{n} d s=0\right\} \quad \forall \varepsilon>0 .
\end{aligned}
$$

Furthemore,

$$
\begin{array}{r}
\left(\left\|\left.\mathbf{u}\right|_{\partial \Omega}\right\|_{L^{2}\left(0, T ; \mathbf{H}^{1}(\partial \Omega)\right)}^{2}+\left\|\left.u_{n}\right|_{\partial \Omega}\right\|_{H^{1-\varepsilon}\left(0, T ; H^{-1}(\partial \Omega)\right)}^{2}+\|\mathbf{u} \boldsymbol{\tau} \mid \partial \Omega\|_{H^{1 / 2}\left(0, T ; L^{2}(\partial \Omega)\right)}^{2}\right) \\
\leq C_{\varepsilon}\|\mathbf{u}\|_{\mathcal{V}^{(3 / 2)}\left(Q_{T}\right)}^{2}
\end{array}
$$

where the constant $C_{\varepsilon}$ depends on $\varepsilon>0$ but does not depend on $\mathbf{u}$.

Theorem 4.5 Assume that a vector field $\mathbf{b}=b_{n}+\mathbf{b}_{\boldsymbol{\tau}}$ belongs to the space

$$
\begin{aligned}
& \left\{\mathbf{z} \in L^{2}\left(0, T ; \mathbf{H}^{1}(\partial \Omega)\right): z_{n} \in H^{1}\left(0, T ; \mathbf{H}^{-1}(\partial \Omega)\right)\right. \\
& \left.\quad \mathbf{z}_{\boldsymbol{\tau}} \in H^{2 / 5}\left(0, T ; \mathbf{H}^{1 / 5}(\partial \Omega)\right), \quad \int_{\partial \Omega} z_{n} d s=0 \quad \text { a.e. } \quad t \in[0, T]\right\} .
\end{aligned}
$$

Then there exists an $\mathbf{u} \in \mathcal{V}^{3 / 2}\left(Q_{T}\right)$ satisfying

$$
\left.\mathbf{u}\right|_{S_{T}}=\mathbf{b}
$$

and the estimate

$\|\mathbf{u}\|_{\mathcal{V}^{(3 / 2)}\left(Q_{T}\right)}^{2} \leq C\left\{\|b\|_{L^{2}\left(0, T ; H^{1}(\partial \Omega)\right.}^{2}+\left\|b_{n}\right\|_{H^{1}\left(0, T ; H^{-1}(\partial \Omega)\right)}^{2}+\left\|b_{\boldsymbol{\tau}}\right\|_{H^{2 / 5}\left(0, T ; H^{1 / 5}(\partial \Omega)\right)}^{2}\right\}$,

where $C$ does not depend on $\mathbf{b}$

Remark. It is easy to show that

$L^{2}\left(0, T ; \mathbf{H}^{1}(\partial \Omega)\right) \cap H^{2 / 5}\left(0, T ; \mathbf{H}^{1 / 5}(\partial \Omega)\right) \subset L^{2}\left(0, T ; \mathbf{H}^{1}(\partial \Omega)\right) \cap H^{1 / 2}\left(0, T ; \mathbf{L}_{2}(\partial \Omega)\right) ;$

thus we see that the trace space (4.13) is narrower than the corresponding space in Theorem 4.4.

Remark. The proof of Theorems 4.4 and 4.5 (in the $3 D$ case) uses the system

$$
\operatorname{curl} \boldsymbol{\psi}=\mathbf{u} \quad \text { in } \Omega, \quad \operatorname{div} \boldsymbol{\psi}=0 \quad \text { in } \Omega, \quad \text { and }\left.\quad(\boldsymbol{\psi} \cdot \mathbf{n})\right|_{\partial \Omega}=0
$$

in place of $\operatorname{curl} \psi=\mathbf{u}$ in the $2 D$-case. The analysis of regularity is much more involved. Details will be published elsewhere.

\subsection{Estimates for the solutions of the Navier-Stokes equations with nonhomogeneous Dirichlet boundary data}

We now consider the boundary value problem for the Navier-Stokes equation in the form introduced in Definition 2.1. The boundary data $\mathbf{b}$ is assumed to 
satisfy the compatibility condition $\int_{\partial \Omega} \mathbf{b} \cdot \mathbf{n} d s=0$. Our goal here is, with the help of the extension theorem (Theorem 4.3), to establish the existence of a solution for (2.2)-(2.4) and derive estimates for the solutions in the space of critical smoothness in terms of the data $\mathbf{w}_{0}$ and $\mathbf{b}$.

Theorem 4.6. Let $\Omega \subset \mathbb{R}^{2}$. Assume that $\mathbf{b}$ and $\mathbf{w}_{0}$ satisfy

$\int_{\partial \Omega} b_{n} d s=0 \quad$ a.e. $t \in[0, T], \quad b_{n} \in L^{2}\left(0, T ; H^{1 / 2}(\partial \Omega)\right) \cap H^{3 / 4}\left(0, T ; H^{-1}(\partial \Omega)\right)$,

$$
\begin{gathered}
b_{\tau} \in L^{2}\left(0, T ; H^{1 / 2}(\partial \Omega)\right) \cap H^{1 / 4}\left(0, T ; L^{2}(\partial \Omega)\right), \\
\mathbf{w}_{0} \in \mathbf{V}^{0}(\Omega)
\end{gathered}
$$

and

$$
\left.\left(\mathbf{w}_{0} \cdot \mathbf{n}\right)\right|_{\partial \Omega}=\left.b_{n}\right|_{t=0} .
$$

Then, there exists a unique solution $\mathbf{w} \in \mathcal{V}^{(1)}\left(Q_{T}\right)$ for the problem (2.1)-(2.3). Moreover, the solution satisfies the estimate

$$
\begin{array}{r}
\|\mathbf{w}\|_{\mathcal{V}^{(1)}\left(Q_{T}\right)}^{2} \leq B\left(\left\|\mathbf{w}_{0}\right\|_{\mathbf{L}^{2}(\Omega)},\left\|b_{n}\right\|_{L^{2}\left(0, T ; H^{1 / 2}(\partial \Omega)\right)}+\left\|b_{n}\right\|_{H^{3 / 4}\left(0, T ; H^{-1}(\partial \Omega)\right)}\right. \\
\left.\left\|b_{\tau}\right\|_{L^{2}\left(0, T ; H^{1 / 2}(\partial \Omega)\right)}+\left\|b_{\tau}\right\|_{H^{1 / 4}\left(0, T ; L^{2}(\partial \Omega)\right)},\left|\mathbf{v}_{\infty}\right|\right)
\end{array}
$$

where $B(\cdot, \cdot, \cdot, \cdot)$ is a continuous positive function defined on $\mathbb{R} \times \mathbb{R} \times \mathbb{R} \times \mathbb{R}$. Proof. Let $\mathbf{u} \in \mathcal{V}^{(1)}\left(Q_{T}\right)$ be the extension of the data $\mathbf{b}$ into $Q_{T}$ constructed in Theorem 4.3. With the change of variable relation $\mathbf{w}=\mathbf{u}+\boldsymbol{\eta}$, it can be easily checked that the existence and uniqueness of a desired $\mathbf{w}$ is equivalent to the existence and uniqueness of a $\boldsymbol{\eta}$ satisfying the equation

$$
\begin{gathered}
\left\langle\partial_{t} \boldsymbol{\eta}(t), \mathbf{z}\right\rangle+\mu \int_{\Omega} \nabla \boldsymbol{\eta}(t): \nabla \mathbf{z} d \mathbf{x}+\int_{\Omega}\left(\left(\boldsymbol{\eta}(t)+\mathbf{u}(t)+\mathbf{v}_{\infty}\right) \cdot \nabla\right) \boldsymbol{\eta}(t) \cdot \mathbf{z} d \mathbf{x} \\
+\int_{\Omega}(\boldsymbol{\eta}(t) \cdot \nabla) \mathbf{u}(t) \cdot \mathbf{z} d \mathbf{x}=\langle\mathbf{f}(t), \mathbf{z}\rangle \quad \forall \mathbf{z} \in \mathbf{V}_{0}^{1}(\Omega), \text { a.e. } t \in(0, T), \\
\left.\boldsymbol{\eta}\right|_{S_{T}}=\mathbf{0} \quad \text { in } L^{2}\left(0, T ; \mathbf{H}^{1 / 2}(\partial \Omega)\right),
\end{gathered}
$$

and

$$
\left.\boldsymbol{\eta}\right|_{t=0}=\boldsymbol{\eta}_{0} \equiv \mathbf{w}_{0}-\left.\mathbf{u}\right|_{t=0} \quad \text { in } \mathbf{V}_{0}^{0}(\Omega)
$$

where

$$
\langle\mathbf{f}(t), \mathbf{z}\rangle=-\mu \int_{\Omega} \nabla \mathbf{u}(t): \nabla \mathbf{z} d \mathbf{x}-\left\langle\partial_{t} \mathbf{u}(t), \mathbf{z}\right\rangle-\int_{\Omega}\left[\left(\mathbf{u}(t)+\mathbf{v}_{\infty}\right) \cdot \nabla\right] \mathbf{u}(t) \cdot \mathbf{z} d \mathbf{x}
$$

The existence and uniqueness of the solution $\boldsymbol{\eta} \in \mathcal{V}^{(1)}\left(Q_{T}\right)$ for (4.18)-(4.20) can be proved in exactly the same way as that for the two-dimensional Navier-Stokes 
equations with homogeneous boundary conditions in exterior domains; see, e.g., [14] or [17]. The proof of the estimate is not difficult also.

Remark. The proof of the $3 D$ version of Theorem 4.6 can be realized for small initial and boundary data, just as in the case of homogeneous boundary value problems. There is also another case where the $3 D$ version of the existence theorem can be proved. That is the case where the initial condition $\mathbf{w}_{0}$ is a steady-state solution of the Navier-Stokes equation (2.2) and boundary data are small enough.

\section{Formulation of optimal control problems and optimality systems.}

From the results of Section 4, we have a very clear picture of what the minimal smoothness should be for the boundary control, i.e., the minimal smoothness should be that of the trace space (see Theorem 4.1 and 4.3-4.5). Naturally, the control should be measured in a norm that is not weaker than the norm for the trace space. For computational convenience, we will strengthen the fractional time derivatives to the first derivative $\partial_{t}$ in the functional. Also, the particular form of the drag functional, i.e., the term $\int_{0}^{T} \int_{\Omega} \mathcal{D}(\mathbf{w}): \mathcal{D}(\mathbf{w}) d \mathbf{x} d t$, implies that in order for $\mathbf{w}$ to belong to the desired trace space, it is sufficient to use the norm

$$
\int_{0}^{T} \int_{\partial \Omega}\left|\partial_{t} \mathbf{w}\right|^{2} d s d t \quad \text { in } 2 D
$$

for the controls. In $3 D$, we have to use the norm

$$
\int_{0}^{T} \int_{\partial \Omega}\left(\left|\partial_{t} \mathbf{w}\right|^{2}+\left|\nabla_{s} \mathbf{w}\right|^{2}\right) d s d t \quad \text { in } 3 D
$$

where $\nabla_{s}$ is the surface gradient operator. Also, we have to include the constraints connected with the term $\int_{0}^{T} \int_{\partial \Omega}\left|\mathbf{w}+\mathbf{v}_{\infty}\right|^{k} d s d t$ for some $k \geq 3$.

Hence, the drag functional (compromized by adding the norm of the control) is chosen to be

$$
\begin{aligned}
\mathcal{J}(\mathbf{w})= & \mu \int_{0}^{T} \int_{\Omega} \mathcal{D}(\mathbf{w}): \mathcal{D}(\mathbf{w}) d \mathbf{x} d t+\frac{\rho}{4} \int_{0}^{T} \int_{\partial \Omega}|\mathbf{w}|^{2}\left(\mathbf{w}+\mathbf{v}_{\infty}\right) \cdot \mathbf{n} d s d t \\
& +\frac{\rho}{4} \int_{\Omega}|\mathbf{w}(T, \mathbf{x})|^{2} d \mathbf{x} \\
& +\rho N\left(\int_{0}^{T} \int_{\partial \Omega}\left|\mathbf{w}+\mathbf{v}_{\infty}\right|^{k} d s d t+\int_{0}^{T} \int_{\partial \Omega}\left|\partial_{t} \mathbf{w}\right|^{2} d s d t\right), \quad \text { in } 2 D
\end{aligned}
$$

and

$$
\begin{aligned}
& \mathcal{J}(\mathbf{w})=\mu \int_{0}^{T} \int_{\Omega} \mathcal{D}(\mathbf{w}): \mathcal{D}(\mathbf{w}) d \mathbf{x} d t+\frac{\rho}{4} \int_{0}^{T} \int_{\partial \Omega}|\mathbf{w}|^{2}\left(\mathbf{w}+\mathbf{v}_{\infty}\right) \cdot \mathbf{n} d s d t \\
& \quad+\frac{\rho}{4} \int_{\Omega}|\mathbf{w}(T, \mathbf{x})|^{2} d \mathbf{x} \\
& \quad+\rho N\left(\int_{0}^{T} \int_{\partial \Omega}\left|\mathbf{w}+\mathbf{v}_{\infty}\right|^{k} d s d t+\int_{0}^{T} \int_{\partial \Omega}\left(\left|\partial_{t} \mathbf{w}\right|^{2}+\left|\nabla_{s} \mathbf{w}\right|^{2}\right) d s d t\right), \text { in } 3 D
\end{aligned}
$$

where $\mathbf{w}=\mathbf{v}-\mathbf{v}_{\infty}, k \geq 3$, and $N>0\left(N>\frac{1}{4}\right.$ if $\left.k=3\right)$. 
Extremal solutions are sought in the space

$$
\begin{aligned}
Y=\left\{\mathbf{w} \in \mathcal{V}^{(1)}\left(Q_{T}\right):\right. & \left.\left(\partial_{t} \mathbf{w}\right)\right|_{\partial \Omega} \in L^{2}\left(0, T ; \mathbf{L}^{2}(\partial \Omega)\right), \\
& \left.\int_{\partial \Omega} \partial_{t} \mathbf{w} \cdot \mathbf{n} d s=0,\left.\quad \mathbf{w}\right|_{\partial \Omega} \in \mathbf{L}^{k}((0, T) \times \partial \Omega)\right\} \quad \text { in } 2 D
\end{aligned}
$$

or

$$
\begin{aligned}
Y=\left\{\mathbf{w} \in \mathcal{V}^{(3 / 2)}\left(Q_{T}\right):\right. & \left.\left(\partial_{t} \mathbf{w}\right)\right|_{\partial \Omega} \in L^{2}\left(0, T ; \mathbf{L}^{2}(\partial \Omega)\right),\left.\left(\nabla_{s} \mathbf{w}\right)\right|_{\partial \Omega} \in L^{2}\left(0, T ; \mathbf{L}^{2}(\partial \Omega)\right), \\
& \left.\int_{\partial \Omega} \partial_{t} \mathbf{w} \cdot \mathbf{n} d s=0,\left.\quad \mathbf{w}\right|_{\partial \Omega} \in \mathbf{L}^{k}((0, T) \times \partial \Omega)\right\} \quad \text { in } 3 D
\end{aligned}
$$

equipped with the norm

$$
\|\mathbf{w}\|_{Y}=\|\mathbf{w}\|_{\mathcal{V}^{(1)}\left(Q_{T}\right)}+\left\|\partial_{t} \mathbf{w}\right\|_{L^{2}\left(0, T ; \mathbf{L}^{2}(\partial \Omega)\right)}+\|\mathbf{w}\|_{\mathbf{L}^{k}((0, T) \times \partial \Omega)}
$$

or

$\|\mathbf{w}\|_{Y}=\|\mathbf{w}\|_{\mathcal{V}^{(3 / 2)}\left(Q_{T}\right)}+\left\|\partial_{t} \mathbf{w}\right\|_{L^{2}\left(0, T ; \mathbf{L}^{2}(\partial \Omega)\right)}+\left\|\nabla_{s} \mathbf{w}\right\|_{L^{2}\left(0, T ; \mathbf{L}^{2}(\partial \Omega)\right)}+\|\mathbf{w}\|_{\mathbf{L}^{k}((0, T) \times \partial \Omega)}$, respectively.

Note that $\mathbf{w} \in Y$ necessarily implies that $\mathbf{w}(0) \in \mathbf{W}$ where

$$
\mathbf{W}=\left\{\mathbf{w} \in \mathbf{V}^{0}(\Omega):\left.(\mathbf{w} \cdot \mathbf{n})\right|_{\partial \Omega} \in H^{1 / 4}(\partial \Omega) \cap L^{1+k / 2}(\partial \Omega)\right\} .
$$

Thus, the specified initial condition in the Navier-Stokes equations must satisfy the regularity condition

$$
\left.\mathbf{w}\right|_{t=0}=\mathbf{w}_{0} \equiv \mathbf{v}_{0}-\mathbf{v}_{\infty} \in \mathbf{W}
$$

We are now in a position to formulate the drag minimization problem.

$2 D$ Optimal Control Problem. Suppose that $\mathbf{w}_{0} \equiv \mathbf{v}_{0}-\mathbf{v}_{\infty} \in \mathbf{W}$. Seek a $\mathbf{w} \in Y$ such that the functional (5.1) is minimized subject to the constraints (2.3) and (2.5), where $k \geq 3$ and $N>0$ with $N>\frac{1}{4}$ if $k=3$.

$3 D$ Optimal Control Problem. Suppose that $\mathbf{w}_{0} \equiv \mathbf{v}_{0}-\mathbf{v}_{\infty} \in \mathbf{W}$. Seek a $\mathbf{w} \in Y$ such that the functional (5.2) is minimized subject to the constraints (2.3) and (2.5), where $k \geq 3$ and $N>0$ with $N>\frac{1}{4}$ if $k=3$.

We now formulate the results for the $2 D$ optimal control problem. We begin from the existence theorem. We need the following lemma:

Lemma 5.1 There exists a constant $C>0$ depending only on $\Omega$ such that

$$
\|\mathbf{u}\|_{\mathbf{H}^{1 / 2}(\partial \Omega)} \leq C\left(\int_{\Omega}|D(\mathbf{u})|^{2} d \mathbf{x}+\int_{\partial \Omega}|\mathbf{u}|^{2} d s\right) \quad \forall \mathbf{u} \in \mathbf{H}^{1}(\Omega) .
$$

Theorem 5.1 There exists a solution $\mathbf{w} \in Y$ for the $2 D$ Optimal Control Problem.

Draft of Proof. Let $\mathbf{w}_{n}$ be a minimizing sequence of $2 D$ Optimal Control Problem. Then $J\left(\mathbf{w}_{n}\right) \leq C<\infty$ and by inequality (5.3) we obtain:

$$
\left\|\partial_{t} \mathbf{w}_{n}\right\|_{L^{2}\left(0, T ; \mathbf{L}^{2}(\partial \Omega)\right)}+\left\|\mathbf{w}_{n}\right\|_{\mathbf{L}^{k}((0, T) \times \partial \Omega)}+\|\mathbf{w}\|_{\mathbf{H}^{1 / 2}((0, T) \times \partial \Omega)} \leq C<\infty .
$$

ESAIM: Proc., Vol. 4, 1998, 97-116 
By this inequality and Theorem 4.6 (this is the main point of proof) we have

$$
\left\|\mathbf{w}_{n}\right\|_{Y} \leq C<\infty
$$

This estimate allows to pass to limit in $\mathbf{w}_{n}$ as $n \rightarrow \infty$ and to finish the Theorem 5.1 proof.

We now derive the weak form of an optimality system for the $2 D$ case.

Theorem 5.2. Assume $\mathbf{w} \in \mathcal{V}^{(1)}\left(Q_{T}\right)$ is a solution for the optimal control problem. Then there exists a $\mathbf{q} \in \mathcal{V}^{(1)}\left(Q_{T}\right) \cap L^{2}\left(0, T ; \mathbf{V}_{0}^{1}(\Omega)\right)$ such that

$$
\begin{aligned}
& 2 \mu \int_{0}^{T} \int_{\Omega} \mathcal{D}(\mathbf{w}): \mathcal{D}(\mathbf{h}) d \mathbf{x} d t+2 \mu \int_{0}^{T} \int_{\Omega} \mathcal{D}(\mathbf{h}): \mathcal{D}(\mathbf{q}) d \mathbf{x} d t \\
& \quad+\int_{0}^{T} \int_{\Omega}\left\{(\mathbf{h} \cdot \nabla) \mathbf{w}+(\mathbf{w} \cdot \nabla) \mathbf{h}+\left(\mathbf{v}_{\infty} \cdot \nabla\right) \mathbf{h}\right\} \cdot \mathbf{q} d \mathbf{x} d t \\
& \quad+\int_{0}^{T}\left\langle\partial_{t} \mathbf{h}(t, \cdot), \mathbf{q}(t, \cdot)\right\rangle d t+N\left(\int_{0}^{T} \int_{\partial \Omega} 2 \partial_{t} \mathbf{w} \cdot \partial_{t} \mathbf{h} d s d t\right. \\
& \left.\quad+k \int_{0}^{T} \int_{\partial \Omega}\left|\mathbf{w}+\mathbf{v}_{\infty}\right|^{k-2}\left(\mathbf{w}+\mathbf{v}_{\infty}\right) \cdot \mathbf{h} d s d t\right) \\
& \quad+\frac{1}{2} \int_{0}^{T} \int_{\partial \Omega}\left\{\left(\mathbf{w}+\mathbf{v}_{\infty}\right) \cdot \mathbf{n}(\mathbf{w} \cdot \mathbf{h})+\frac{1}{2}(\mathbf{h} \cdot \mathbf{n})|\mathbf{w}|^{2}\right\} d s d t \\
& \quad+\frac{1}{2} \int_{\Omega} \mathbf{w}(T, \mathbf{x}) \cdot \mathbf{h}(T, \mathbf{x}) d \mathbf{x}=0 \quad \forall \mathbf{h} \in Y_{0}
\end{aligned}
$$

where $Y_{0} \equiv\left\{\mathbf{y} \in Y:\left.\mathbf{y}\right|_{t=0}=\mathbf{0}\right\}$.

Proof. In the functional (5.1), the differentiability of $\mathcal{G}$ is clearly satisfied. We recall from Section 3 the definitions of $X_{1}=Y_{0}, X_{2}=L^{2}\left(0, T ; \mathbf{V}^{-1}(\Omega)\right)$, $f: X_{1} \rightarrow \mathbb{R}$ and $F: X_{1} \rightarrow X_{2}$. We also recall that to show $F^{\prime}(\mathbf{0})$ is an epimorphism, we have reduced to show that the system (3.4)-(3.6) has a solution for every given right-hand side $\mathbf{f}$. We may prove as well as Theorem 4.6 that (3.4)-(3.6) indeed has a (unique) solution $\mathbf{y} \in \mathcal{V}^{(1)}\left(Q_{T}\right)$ (the situation now is even simpler than the case of Theorem 4.6 as the system (3.4)-(3.6) is linear). Clearly, $\mathbf{y} \in Y_{0}$. Hence, we have verified all the assumption in Theorem 3.1 and we conclude that there exists a $\mathbf{q} \in X_{2}^{*}=L^{2}\left(0, T ; \mathbf{V}_{0}^{1}(\Omega)\right)$ such that $\left.\left\langle\mathcal{L}_{\mathbf{y}}(\mathbf{y}, \mathbf{q}), \mathbf{h}\right\rangle\right|_{y=0}=0$ for all $\mathbf{h} \in Y_{0}$. This equality implies (5.4).

We may formally derive the following strong form of the optimality system: the partial differential equations (in the sense of distributions)

$$
\begin{aligned}
& \partial_{t} \mathbf{w}-\mu \Delta \mathbf{w}+\left(\left(\mathbf{w}+\mathbf{v}_{\infty}\right) \cdot \nabla\right) \mathbf{w}+\nabla p=\mathbf{0} \quad \text { in }(0, T) \times \Omega \\
& \nabla \cdot \mathbf{w}=0 \quad \text { in }(0, T) \times \Omega, \\
& -\partial_{t} \mathbf{q}-\mu \Delta \mathbf{q}+\mathbf{q} \cdot(\nabla \mathbf{w})^{T}-(\mathbf{w} \cdot \nabla) \mathbf{q}-\left(\mathbf{v}_{\infty} \cdot \nabla\right) \mathbf{q}+\nabla r=\mu \Delta \mathbf{w}-\nabla p \\
& \nabla \cdot \mathbf{q}=0 \quad \text { in }(0, T) \times \Omega
\end{aligned}
$$

the initial and terminal conditions

$$
\begin{aligned}
& \mathbf{w}(0, \cdot)=\mathbf{w}_{0}(\cdot) \quad \text { in } \mathbf{V}^{0}(\Omega) \\
& \mathbf{q}(T, \cdot)+\frac{1}{2} \mathbf{w}_{\sigma}(T, \cdot)=0 \quad \text { in } \mathbf{V}_{0}^{0}(\Omega)
\end{aligned}
$$

where $\mathbf{w}_{\sigma}(T, \cdot)$ is the projection of $\mathbf{w}(T, \cdot)$ onto $\mathbf{V}_{0}^{0}(\Omega)$; the (lateral) boundary conditions

$$
\begin{aligned}
& \left.\mathbf{q}\right|_{S_{T}}=\mathbf{0}, \\
& 2 N \partial_{t t}^{2}(\gamma \mathbf{w})-\mathcal{A}(\mathbf{w})-\mathcal{T}(\mathbf{w}, p) \mathbf{n}-\mathcal{T}(\mathbf{q}, r) \mathbf{n}=\eta(t) \mathbf{n},
\end{aligned}
$$


where

$$
\begin{aligned}
& \mathcal{T}(\mathbf{w}, p)=-p I+2 \mu \mathcal{D}(\mathbf{w}) \text { and } \mathcal{T}(\mathbf{q}, r)=-r I+2 \mu \mathcal{D}(\mathbf{q}) \\
& \mathcal{A}(\mathbf{w})=\gamma\left(k N\left|\mathbf{w}+\mathbf{v}_{\infty}\right|^{k-2}\left(\mathbf{w}+\mathbf{v}_{\infty}\right)+\frac{1}{2}\left(\left(\mathbf{w}+\mathbf{v}_{\infty}\right) \cdot \mathbf{n}\right) \mathbf{w}+\frac{|\mathbf{w}|^{2}}{4} \mathbf{n}\right) \\
& \eta(t)=-\int_{\partial \Omega} \mathcal{A}(\mathbf{w}) \cdot \mathbf{n} d s / \int_{\partial \Omega} d s ;
\end{aligned}
$$

and the compatibility conditions

$$
\begin{aligned}
& {\left.[(\gamma \mathbf{w}) \cdot \mathbf{n}]\right|_{t=0}=\left(\gamma \mathbf{w}_{0}\right) \cdot \mathbf{n},} \\
& \left.\left(\partial_{t} \gamma \mathbf{w}\right) \cdot \boldsymbol{\tau}\right|_{t=T}=0 \quad \text { and }\left.\quad\left(\frac{1}{2} \gamma w_{\pi}+2 N \partial_{t} \gamma \mathbf{w} \cdot \mathbf{n}\right)\right|_{t=T}=0,
\end{aligned}
$$

where $\boldsymbol{\tau}$ is the unit tangential along $\partial \Omega$ and $w_{\pi}(t, \cdot)$ is the primitive function of the projection of $\mathbf{w}(t, \cdot)$ onto $\nabla H_{\pi}$ determined by the condition

$$
\int_{\partial \Omega} w_{\pi}(t, \cdot) d s=0 .
$$

The rigorous interpretations of the differential equations, boundary conditions and compatibility conditions were discussed in [9]. In particular, the boundary stresses belong to suitable Besov spaces.

\section{References}

[1] R. Adams, Sobolev Spaces, Academic, New York, 1975.

[2] V. Alekseev, V. Tikhomirov, and S. Fomin, Optimal Control, Consultants Bureau, New York, 1987.

[3] H. Fattorini and S. Sritharan, Existence of optimal controls for viscous flow problems, to appear.

[4] H. Fattorini and S. SRitharan, Necessary and sufficient conditions for optimal controls in viscous flow problems, to appear.

[5] H. Fattorini and S. Sritharan, Optimal control theory for viscous flow problems, to appear.

[6] A.V. Fursikov, Control problems and theorems conserning the unique solvability of a mixed boundary value problem for the three-dimensional NavierStokes and Euler equations, Math USSR Sbornik, 43 (2), (1982), p.p.251273.

[7] A.V. Fursikov, Propierties of solutions of certain extremal problems connected with the Navier-Stokes equations, Math. USSR Sbornik, 46 (3), (1983), p.p.323-351.

[8] A.V. Fursikov, Certain otpimal control problems for Navier-Stokes system with distributed control function, IMA Preprints series \#1348, October 1995.

ESAIM: Proc., Vol. 4, 1998, 97-116 
[9] A.V. Fursikov, M.D. Gunzburger and L.S. Hou, Boundary value problems and optimal boundary control for the Navier-Stokes system: the twodimensional case, SIAM J. Control Opt., to appear.

[10] V. Girault and P.-A. Raviart, Finite Element Methods for Navier-Stokes Equations, Springer, Berlin, 1986.

[11] M. Gunzburger, L. Hou, and T. Svobodny, Analysis and finite element approximation of optimal control problems for the stationary Navier-Stokes equations with Dirichlet controls, Modél. Math. Anal. Numér., 25 (1991), pp. $711-748$.

[12] M. Gunzburger, L. Hou, and T. Svobodny, Boundary velocity control of incompressible flow with an application to viscous drag reduction, SIAM J. Control Opt., 30 (1992), pp. 167-181.

[13] L. Hou and T. Svobodny, Optimization problems for the Navier-Stokes equations with regular boundary controls, J. Math. Anal. Appl., 177 (1993), pp. $342-367$.

[14] O. Ladyzhenskaya, The Mathematical Theory of Viscous Incompressible Flow, Gordon and Breach, New York, 1963.

[15] J.-L. Lions Contrôle des systèmes distribués singulier, Gauthier-Villars, Paris, 1983.

[16] J.-L. Lions and E. Magenes, Non-Homogeneous Boundary Value Problems and Applications I, Springer-Verlag, Berlin, 1972.

[17] R. Temam, Navier-Stokes Equations, North-Holland, Amsterdam, 1979. 